Cahiers $d u$ MONDE RUSSE

\section{Cahiers du monde russe}

Russie - Empire russe - Union soviétique et États indépendants

$52 / 4 \mid 2011$

Varia

\title{
Tracy Dennison, The Institutional Framework of Russian Serfdom
}

\section{Alessandro Stanziani}

\section{OpenEdition}

Journals

Édition électronique

URL : http://journals.openedition.org/monderusse/7521

DOI : 10.4000/monderusse.7521

ISSN : 1777-5388

\section{Éditeur}

Éditions de l'EHESS

\section{Édition imprimée}

Date de publication : 20 décembre 2011

Pagination : 700-702

ISBN : 978-2-7132-2353-2

ISSN : 1252-6576

\section{Référence électronique}

Alessandro Stanziani, «Tracy Dennison, The Institutional Framework of Russian Serfdom », Cahiers du monde russe [En ligne], 52/4 | 2011, mis en ligne le 28 novembre 2012, Consulté le 25 septembre 2020. URL : http://journals.openedition.org/monderusse/7521; DOI : https://doi.org/10.4000/monderusse. 7521

Ce document a été généré automatiquement le 25 septembre 2020.

(c) École des hautes études en sciences sociales 


\title{
Tracy Dennison, The Institutional Framework of Russian Serfdom
}

\author{
Alessandro Stanziani
}

\section{RÉFÉRENCE}

Tracy DENNISON, The Institutional Framework of Russian Serfdom. Cambridge :

Cambridge University Press, 2011, XX +254 p.

1 Voici un ouvrage attendu, fruit d'une longue gestation, l'auteur ayant commencé à publier ses travaux sur le servage en 2003. C'est un ouvrage qui entre dans un double sillage : celui de l'histoire économique dite néo-institutionnaliste (visant à étudier les marchés à partir des institutions sous-jacentes) et l'intérêt renouvelé pour le « deuxième servage » en Europe centrale et orientale. Dans un cas comme dans l'autre, le « cas russe » étant singulièrement absent des études existantes à quelques exceptions près, cet ouvrage constitue un excellent départ en vue de combler cette lacune. Son argument principal est simple: pendant des générations, le mythe de la commune paysanne et du paysan autosuffisant a dominé les études sur le monde rural russe. Il est temps de dépasser ce mythe et, avec lui, l'exceptionnalité présumée de la Russie. À cette fin, l'auteur considère justement qu'il est nécessaire d'examiner de très près le fonctionnement réel du servage, à l'échelle d'un domaine, en l'occurrence celui des Šeremetev à Voščažnikovo, dans la province de Jaroslav (régions industrielles centrales). Dans les différents chapitres de l'ouvrage, Tracy Dennison fournit ainsi une image extrêmement détaillée de la vie dans le domaine en question : environnement, population, mariages, activités économiques (crédit, terre, travail), déplacements, etc. L'image qui en ressort n'est pas conforme aux lieux communs sur la Russie. Le servage se réduit à un ensemble d'obligations, prévues dans les instructions des seigneurs pour l'administration de leurs domaines, mais qui, dans la réalité, peuvent se contourner par un système qu'on pourrait qualifier d'extorsion institutionnalisée. En effet, il suffit de payer une taxe au seigneur pour avoir le droit de se marier hors du domaine, de faire du commerce, de travailler en ville, d'échapper à la conscription, et ainsi de suite. Plus 
étonnant encore, les paysans achètent des terres à titre individuel ou familial, même si leurs droits de propriété demeurent imparfaits car soumis au bon vouloir du seigneur. Dans ce cadre, la commune apparaît moins égalitaire et contraignante qu'on l'affirme d'habitude, car elle finit par encourager une certaine stratification sociale et la différenciation dans les activités économiques et dans l'accès à la propriété. Selon l'auteur, ces éléments réunis expliquent le dynamisme des campagnes russes, même avant l'abolition du servage, mais aussi ses limites. Même si les institutions russes paraissent ainsi plus souples qu'on l'affirme habituellement, elles limitent néanmoins le développement économique, en particulier en restreignant le plein accès à la propriété.

2 Nous aurions envie de lire cet ouvrage comme on le faisait jadis pour les meilleurs travaux publiés à l'époque soviétique: si nous mettons de côté l'introduction et les têtes de chapitre, bourrées de références obligées, avec souvent peu de lien avec le contenu qui suit, le cœur de l'ouvrage constitue une mine d'information et offre dès lors un aperçu assez novateur du servage russe. Les problèmes surgissent au moment où, dans l'introduction surtout, l'auteur essaie d'aller plus loin et de monter en généralité. Elle part du constat, tout à fait correct, que le servage russe n'est pas une institution uniforme, mais un ensemble de pratiques finalement assez différentes d'un domaine à l'autre. Comme d'autres auteurs avant elle (Hoch, Czap, Melton) ${ }^{1}$, cette posture permet de valoriser une étude centrée sur un seul domaine, et de nuancer la critique immédiate qui consisterait à mettre en cause le caractère significatif et la représentativité du domaine choisi, et donc de cette étude. Car, si le servage change d'un domaine à l'autre, alors, effectivement, nous sommes obligés d'attendre la multiplication de ces études avant de pouvoir avancer des conclusions plus générales.

Cependant l'auteur finit par affaiblir cette posture en cherchant à accorder une valeur générale à son étude. Non seulement le titre (le contexte institutionnel du servage russe et non pas du servage à Voščǎnikovo), mais aussi l'introduction et les conclusions visent cette portée plus générale. C'est en particulier évident lorsque Dennison souligne le caractère dynamique du servage russe et lorsqu'elle critique le " mythe paysan ». Ce dernier est associé à la théorie de Čajanov, mais aussi à toute la tradition populiste et néo-populiste russe qui, selon l'auteur, aurait finalement influencé l'ensemble de l'historiographie soviétique et occidentale. C'est pousser un peu loin le bouchon. Car, au fond, l'approche čajanovienne et le «mythe paysan » en général se sont confrontés, dans la Russie tsariste déjà, aux analyses occidentalistes, puis marxistes, proposant une image tout à fait autre des campagnes russes, bien plus proches, suivant ces courants, des dynamiques à l'œuvre dans d'autres pays européens. Ensuite, à l'époque soviétique, ce même mythe a été vite censuré et il est resté dans l'ombre jusqu'à la perestroïka. Quant à l'historiographie occidentale, on a également l'impression que, en ce cas aussi, l'auteur exagère l'emprise de l'approche čajanovienne et qu'elle rapproche des auteurs du seul fait qu'ils étudient les aspects ruraux, alors qu'ils sont souvent plus éloignés les uns des autres qu'elle ne le dit (Hoch, Shanin, Confino). Bref, le "mythe paysan" a sans doute été important, mais moins que Dennison l'affirme et, inversement, l'argumentaire de cette dernière n'est pas tout à fait nouveau. Au fond, toutes les analyses menées en Russie au tournant du siècle, puis par la suite, et qui se focalisaient sur les régions industrielles comme Jaroslav au cœur de l'étude de Dennison, avaient mis en évidence le dynamisme des communes paysannes et leur intégration dans les marchés. Par la suite, à l'époque soviétique, 
d'autres travaux avaient produit ce même résultat pour le $\mathrm{XVIII}^{\mathrm{e}}$ et le début du $\mathrm{XIX}^{\mathrm{e}}$ siècle. Du coup, il paraît difficile d'opposer, comme le fait Dennison, ses conclusions à celles de tous les auteurs ayant analysé les campagnes russes car, tout simplement, les résultats dépendent de la région étudiée. Les domaines des Terres noires centrales affichent un comportement beaucoup plus proche du "mythe paysan», par comparaison avec des domaines comme celui qui est au cœur de l'ouvrage de Dennison. Et cet aspect avait malgré tout été parfaitement compris et analysé même par Čajanov et maints autres auteurs du tournant du siècle.

4 La question finalement demeure : afin de bien comprendre le fonctionnement d'une société, il n'est plus possible de se fier uniquement à des données de synthèse, comme celles des zemstva, par exemple; en revanche, la dimension locale et archivistique est désormais indépassable. À partir de là, deux voies s'ouvrent devant nous : soit nous attendons la multiplication des études locales avant de tirer des conclusions (mais encore faudra-t-il savoir à partir de quand ces résultats pourront être considérés comme «significatifs» et acceptables); soit nous raisonnons comme les microhistoriens italiens et admettons que, dans le choix de l'objet historique, c'est moins la masse d'informations qui compte, que la pertinence de la question posée. Quelle que soit la direction que prendra l'historiographie, dans un cas comme dans l'autre, l'ouvrage de Tracy Dennison constituera une référence obligée.

\section{NOTES}

1. Steven L. Hoch, Serfdom and Social Control in Russia: Petrovskoe, a Village in Tambov, Chicago : The University of Chicago Press, 1986 ; Peter Czap, «Peasant-Class Courts and Peasant Customary Justice in Russia, 1861-1912 », Journal of Social History, 1 (2), 1967, p. 149-178 ; Edgar Melton, «Enlightened Seigniorialism and Its Dilemmas in Serf Russia, 1750-1830 », Journal of Modern History, 62 (4), 1990, p. 675-708. 